Supporting Information of

\title{
Exact analytical solution of the ground state hydrogenic problem with soft Coulomb potential
}

\author{
Chen Li \\ Beijing National Laboratory for Molecular Sciences, College of Chemistry and Molecular \\ Engineering, Peking University, Beijing 100871, China
}

(Dated: May 22, 2021)

\section{Contents}

I. Asymptotic analysis of ground state energy in the large $Z$ limit $\quad \mathrm{S} 1$

II. Asymptotic analysis of ground state energy in the large $a$ limit

III. Asymptotic analysis of ground state energy in the small $a$ limit for 1D

\section{ASYMPTOTIC ANALYSIS OF GROUND STATE ENERGY IN THE LARGE $Z$ LIMIT}

In the main text, we have derived the relation between the sum of $u_{k}$ and the total energy $E$ as the following,

$$
f_{2}(1)\left(\sum_{k=0}^{\infty} u_{k}\right)^{2}=E
$$

Moreover,

$$
E=f_{3}^{0} u_{0}+f_{4}^{0}
$$

We note that

$$
E=\langle\phi|\hat{T}+\hat{V}| \phi\rangle>\langle\phi|\hat{V}| \phi\rangle=\left\langle\phi\left|-\frac{Z}{\sqrt{a^{2}+r^{2}}}\right| \phi\right\rangle>\left\langle\phi\left|-\frac{Z}{a}\right| \phi\right\rangle=-\frac{Z}{a} .
$$

Combining Eq. (S2) and Eq. (S3) and substituting the expression of $f_{3}^{0}$ and $f_{4}^{0}$, we deduce that $u_{0}<0$. To analyze the asymptotic behavior of $E$, let us divide Eq. (S1) by $Z^{2}$ and reformulate it using Eq. (S2) as,

$$
f_{2}(1)\left(\frac{1}{Z} \sum_{k=0}^{\infty} u_{k}\right)^{2}=\frac{1}{Z}\left(\frac{f_{3}^{0} u_{0}}{Z}+\frac{f_{4}^{0}}{Z}\right) .
$$


In the large $Z$ limit, let us expand $\frac{u_{0}(Z)}{Z}$ in the following convergent series of $Z$,

$$
\frac{u_{0}}{Z}=\sum_{k=1}^{\infty} C_{k}\left(\frac{1}{Z^{p}}\right)^{k}
$$

Here $p>0$ and we note that $\frac{u_{0}}{Z}$ can only contain negative powers of $Z$. Otherwise, because each $u_{k}$ is a polynomial of $u_{0}$ of order $k+1$ (by the recursive relations of $u_{k}$ ), the two sides of Eq. (S4) cannot give identical series of $Z$. Then the leading term of the right hand side (RHS) of Eq. (S4) is $\frac{f_{4}^{0}}{Z^{2}}=-\frac{1}{a Z}$. Comparing with its left hand side (LHS), we deduce that $\frac{1}{Z} \sum_{k=0}^{\infty} u_{k}$ expanded in terms of $\frac{1}{Z^{p}}$ gives no zeroth order term, and the leading term must be on the order $\frac{1}{Z^{p}}$. Thus, we have $\left(\frac{1}{Z^{p}}\right)^{2}=\frac{1}{Z}$, suggesting that $p=\frac{1}{2}$. Therefore, we can rewrite the series expansion of $u_{0}$ as

$$
u_{0}=C_{1} Z^{1 / 2}+C_{2}+C_{3} Z^{-1 / 2}+\cdots .
$$

Using the recursive relation and truncating to $\mathcal{O}(1)$ terms,

$$
\begin{aligned}
u_{1} & =-\frac{1}{f_{1}^{1}+f_{3}^{0}}\left(f_{2}^{1} u_{0}^{2}+f_{3}^{1} u_{0}+f_{4}^{1}\right) \\
& =-\frac{1}{f_{1}^{1}+f_{3}^{0}}\left(f_{2}^{1}\left(C_{1}^{2} Z+2 C_{1} C_{2} Z^{1 / 2}\right)+f_{3}^{1} C_{1} Z^{1 / 2}+\frac{1}{a} Z+\mathcal{O}(1)\right) .
\end{aligned}
$$

We note that the leading term of $u_{k}$ can be at most on the order $Z^{1 / 2}$. Otherwise, $u_{k+1}$ with the ingredient of $u_{0} u_{k}$ in its recursive formula leads to $\mathcal{O}(Z)$ terms, and $u_{k+2}$ with the ingredient of $u_{0} u_{k+1}$ leads to $\mathcal{O}\left(Z^{3 / 2}\right)$ terms, and so on. This ultimately makes the LHS of Eq. (S4) a divergent series of $Z$. To stop the increase of higher powers of $Z$, one has to eliminate the $\mathcal{O}(Z)$ terms in $u_{1}$. This leads to the condition $-\frac{1}{f_{1}^{1}+f_{3}^{0}}\left(f_{2}^{1} C_{1}^{2}+\frac{1}{a}\right)=0$, from which we can solve for $C_{1}= \pm \sqrt{-\frac{1}{a f_{2}^{1}}}= \pm \sqrt{\frac{1}{a}}$. Because $u_{0}$ is negative for all cases, its leading order coefficient $C_{1}$ is also negative. Thus, $C_{1}=-\sqrt{\frac{1}{a}}$. And $u_{1}$ reduces to

$$
u_{1}=-\frac{1}{f_{1}^{1}+f_{3}^{0}}\left(\left(2 f_{2}^{1} C_{1} C_{2}+f_{3}^{1} C_{1}\right) Z^{1 / 2}+\mathcal{O}(1)\right) .
$$

We next analyze the expression for $u_{2}$,

$$
u_{2}=-\frac{1}{2 f_{1}^{1}+f_{3}^{0}}\left(\sum_{l=0}^{1} f_{2}^{2-l} \sum_{j=0}^{l} u_{j} u_{l-j}+\sum_{l=0}^{1} f_{3}^{2-l} u_{l}+\sum_{l=1}^{1} l f_{1}^{2} u_{l}+f_{4}^{2}\right) .
$$

The potentially problematic terms come from $u_{0}^{2}$ and $u_{0} u_{1}$, which we collect as follows,

$$
\begin{aligned}
& -\frac{1}{2 f_{1}^{1}+f_{3}^{0}}\left(f_{2}^{2} u_{0}^{2}+2 f_{2}^{1} u_{0} u_{1}\right) \\
& \rightarrow-\frac{1}{2 f_{1}^{1}+f_{3}^{0}}\left[f_{2}^{2} C_{1}^{2} Z+2 f_{2}^{1} C_{1}\left(-\frac{1}{f_{1}^{1}+f_{3}^{0}}\left(2 f_{2}^{1} C_{1} C_{2}+f_{3}^{1} C_{1}\right)\right) Z\right] .
\end{aligned}
$$


For the same reason as for $u_{1}, \mathcal{O}(Z)$ terms must cancel, which leads to

$$
f_{2}^{2} C_{1}^{2}+2 f_{2}^{1} C_{1}\left(-\frac{1}{f_{1}^{1}+f_{3}^{0}}\left(2 f_{2}^{1} C_{1} C_{2}+f_{3}^{1} C_{1}\right)\right)=0
$$

We can solve for $C_{2}=\frac{1}{2 f_{2}^{1}}\left[\frac{f_{2}^{2}\left(f_{1}^{1}+f_{3}^{0}\right)}{2 f_{2}^{1}}-f_{3}^{1}\right]=\frac{3(n+2)}{16 a}$. Here $n$ is the dimension of the problem and we have used $f_{3}(z)=-\frac{1}{2 a}\left((1-z)^{3}+(n-1)(1-z)\right)$. One can keep this process and evaluate all the $C_{k}$. Finally, we substitute Eq. (S6) into Eq. (S2) and obtain

$$
E=f_{4}^{0}+f_{3}^{0} C_{1} \sqrt{Z}+f_{3}^{0} C_{2}+\mathcal{O}\left(\frac{1}{\sqrt{Z}}\right) .
$$

Substituting $f_{i}^{j}$ in the energy expression, we have the following asymptotic series for the $n$ dimensional problem,

$$
E=-\frac{1}{a} Z+\frac{n}{2 a \sqrt{a}} \sqrt{Z}-\frac{3 n(n+2)}{32 a^{2}}+\mathcal{O}\left(\frac{1}{\sqrt{Z}}\right) .
$$

\section{ASYMPTOTIC ANALYSIS OF GROUND STATE ENERGY IN THE LARGE a LIMIT}

Let us scale the coordinates by $\boldsymbol{\xi}=\frac{1}{a} \mathbf{r}$ and multiply our original Schrödinger equation by $a^{2}$. We have

$$
-\frac{1}{2} \nabla_{\boldsymbol{\xi}}^{2} \phi-\frac{a Z}{\sqrt{1+\xi^{2}}} \phi=a^{2} E \phi .
$$

Eq. (S14) is still a soft Coulomb problem, but with scaled nuclear charge and total energy. As Eq. (S14) suggests, $a^{2} E$ depends on the product of $a$ and $Z$ rather than each of them separately.

By Eq. (S13), we have

$$
a^{2} E=-a Z+\frac{n}{2} \sqrt{a Z}-\frac{3 n(n+2)}{32}+a^{2} \mathcal{O}\left(\frac{1}{\sqrt{Z}}\right) .
$$

$a^{2} E$ is a function of $a Z$ if and only if $a^{2} \mathcal{O}\left(\frac{1}{\sqrt{Z}}\right)=\mathcal{O}\left(\frac{1}{\sqrt{a Z}}\right)$. Thus, in the large $a$ limit we have the following asymptotic expansion,

$$
E=-\frac{1}{a} Z+\frac{n}{2 a \sqrt{a}} \sqrt{Z}-\frac{3 n(n+2)}{32 a^{2}}+\mathcal{O}\left(\frac{1}{a^{5 / 2}}\right) .
$$




\section{ASYMPTOTIC ANALYSIS OF GROUND STATE ENERGY IN THE SMALL a LIMIT FOR 1D}

The asymptotic behavior of the energy in the small a limit for $1 \mathrm{D}$ is much more complicated than the large $a$ limit. As $a \rightarrow 0$, using recursive relations one can show that $u_{k} \rightarrow-\frac{2}{k}$ for large $k$. This has two consequences. First, by Eq. (S1) one find$\mathrm{s} E=-\frac{1}{2}\left(\sum_{k=0}^{\infty} u_{k}\right)^{2} \rightarrow-\infty$. By contrast, for $2 \mathrm{D}$, one can show that $u_{k} \rightarrow-\frac{1}{2^{k}}$, and therefore $E=-\frac{1}{2}\left(\sum_{k=0}^{\infty} u_{k}\right)^{2} \rightarrow-\frac{1}{2}\left(\sum_{k=0}^{\infty}-\frac{1}{2^{k}}\right)^{2}=-2$. This explains the drastically different energy limit in different dimensions. Second, for sufficiently small $a$ in the 1D case, the convergence of $u_{k}$ becomes extremely slow. For $a=10^{-4}$, we need thousands of $u_{k}$ in the iteration to obtain an accurate ground state energy. For smaller $a$, we need even more $u_{k}$.

In the analysis of the asymptotic behavior of the energy for $1 \mathrm{D}$, it turns out difficult to repeat the strategy that we used for the large $a$ or $Z$ limit. Here we adopt a different strategy, namely, the variational approach. As $E \rightarrow-\infty, \alpha=\sqrt{-2 E} \rightarrow \infty$ and $\gamma=\frac{Z}{\alpha} \rightarrow 0$. Neglecting $\gamma$ and the contribution of the modulator function $F$ (they have minor effect in the small $a$ limit), we make the variational ansatz that

$$
\phi=e^{-\alpha \sqrt{a^{2}+x^{2}}}
$$

The variational energy is given by

$$
E[\phi]=\frac{\langle\phi|\hat{T}+\hat{V}| \phi\rangle}{\langle\phi \mid \phi\rangle}
$$

For hydrogen atom, $Z=1$. Using variable transformation $t=\frac{\sqrt{a^{2}+x^{2}}}{a}$, we can rewrite the expectation values in terms of integrals of $t$ as follows,

$$
\begin{aligned}
\langle\phi|\hat{T}| \phi\rangle & =-\frac{1}{2} \int_{-\infty}^{\infty} \phi(x) \frac{d^{2}}{d x^{2}} \phi(x) d x=\alpha^{2} a \int_{1}^{\infty} e^{-2 \alpha a t} \frac{\sqrt{t^{2}-1}}{t} d t, \\
\langle\phi|\hat{V}| \phi\rangle & =-\int_{-\infty}^{\infty} \frac{1}{\sqrt{x^{2}+a^{2}}}|\phi(x)|^{2} d x=-2 \int_{1}^{\infty} e^{-2 \alpha a t} \frac{1}{\sqrt{t^{2}-1}} d t \\
\langle\phi \mid \phi\rangle & =\int_{-\infty}^{\infty}|\phi(x)|^{2} d x=2 a \int_{1}^{\infty} e^{-2 \alpha a t} \frac{t}{\sqrt{t^{2}-1}} d t .
\end{aligned}
$$

The last two integrals can be further expressed in terms of K-Bessel functions as

$$
\begin{gathered}
\langle\phi|\hat{V}| \phi\rangle=-2 K_{0}(2 a \alpha), \\
\langle\phi \mid \phi\rangle=2 a K_{1}(2 a \alpha) .
\end{gathered}
$$


Here $K_{n}(x)$ denotes the $n$th order K-Bessel function. The kinetic energy cannot be written in terms of simple special functions. Let us denote $T(\alpha) \equiv\langle\phi|\hat{T}| \phi\rangle$. Here we use the expansion $\frac{\sqrt{t^{2}-1}}{t}=1-\frac{1}{2 t^{2}}-\frac{1}{8 t^{4}}+\cdots$ and rewrite $T(\alpha)$ and $T^{\prime}(\alpha)=\frac{d T}{d \alpha}$ as

$$
\begin{aligned}
T(\alpha) & =\alpha^{2} a \int_{1}^{\infty} e^{-2 \alpha a t} \frac{\sqrt{t^{2}-1}}{t} d t \\
& =\alpha^{2} a \int_{1}^{\infty} e^{-2 \alpha a t} d t-\alpha^{2} a \int_{1}^{\infty} e^{-2 \alpha a t} \frac{1}{2 t^{2}} d t-\alpha^{2} a \int_{1}^{\infty} e^{-2 \alpha a t} \frac{1}{8 t^{4}} d t+\cdots \\
& =\frac{1}{2} \alpha e^{-2 \alpha a}(1+\mathcal{O}(a \alpha)), \\
T^{\prime}(\alpha) & =2 \alpha a \int_{1}^{\infty} e^{-2 \alpha a t} \frac{\sqrt{t^{2}-1}}{t} d t-2 \alpha^{2} a^{2} \int_{1}^{\infty} e^{-2 \alpha a t} \sqrt{t^{2}-1} d t \\
& =e^{-2 \alpha a}(1+\mathcal{O}(a \alpha))-a \alpha K_{1}(2 a \alpha) .
\end{aligned}
$$

Here we have assumed that $a \alpha<<1$ as $a \rightarrow 0$. Plugging Eqs. (S22)-(S23) into Eq. (S18), the energy as a function of $\alpha$ is given by

$$
E(\alpha)=\frac{T(\alpha)-2 K_{0}(2 a \alpha)}{2 a K_{1}(2 a \alpha)}
$$

The variational minimum is achieved when $E^{\prime}(\alpha)=0$, which gives

$$
K_{1}(2 a \alpha)\left[T^{\prime}(\alpha)+2 a K_{1}(2 a \alpha)\right]+a\left(K_{0}(2 a \alpha)+K_{2}(2 a \alpha)\right)\left[T(\alpha)-2 K_{0}(2 a \alpha)\right]=0 .
$$

The asymptotic expansion of K-Bessel functions are

$$
\begin{aligned}
& K_{0}(2 b)=-\gamma_{0}-\ln b+\mathcal{O}\left(b^{2} \ln b\right), \\
& K_{1}(2 b)=\frac{1}{2 b}+\mathcal{O}(b \ln b) \\
& K_{2}(2 b)=\frac{1}{2 b^{2}}-\frac{1}{2}+\mathcal{O}\left(b^{2} \ln b\right) .
\end{aligned}
$$

Here we have denoted $b=a \alpha$; and $\gamma_{0}$ is the Euler constant. Substituting Eqs. (S28)-(S30) and Eqs. (S24)-(S25) into Eq. (S27), we have

$$
\begin{aligned}
& \left(\frac{1}{b}+\mathcal{O}(b \ln b)\right)\left[e^{-2 b}(1+\mathcal{O}(b))-\frac{1}{2}+\frac{1}{\alpha}\right] \\
& +\left(-\gamma_{0}-\ln b+\frac{1}{2 b^{2}}-\frac{1}{2}+\mathcal{O}\left(b^{2} \ln b\right)\right)\left[\frac{1}{2} b e^{-2 b}(1+\mathcal{O}(b))+\frac{2 b}{\alpha}\left(\gamma_{0}+\ln b\right)\right]=0
\end{aligned}
$$

Keeping only the leading order terms, we arrive at

$$
\frac{1}{b}\left(\frac{1}{2}+\frac{1}{\alpha}\right)+\frac{1}{2 b^{2}} \frac{2 b}{\alpha}\left(\gamma_{0}+\ln b\right)=0
$$


Substituting $b=a \alpha$ back into Eq. (S32), we arrive at a fixed point equation for $\alpha$,

$$
\alpha=-2 \ln a \alpha-2-2 \gamma_{0}=\ln \frac{1}{a^{2} \alpha^{2}}-2-2 \gamma_{0} .
$$

The solution can be approached iteratively,

$$
\begin{aligned}
& \alpha_{0}=\ln \frac{1}{a^{2}}-2-2 \gamma_{0}, \\
& \alpha_{1}=\ln \frac{1}{a^{2} \alpha_{0}^{2}}-2-2 \gamma_{0} \approx \ln \frac{1}{a^{2}\left(\ln \frac{1}{a^{2}}\right)^{2}}-2-2 \gamma_{0}, \\
& \alpha_{2}=\ln \frac{1}{a^{2} \alpha_{1}^{2}}-2-2 \gamma_{0} \approx \ln \frac{1}{a^{2}\left(\ln \frac{1}{a^{2}\left(\ln \frac{1}{a^{2}}\right)^{2}}\right)^{2}}-2-2 \gamma_{0},
\end{aligned}
$$

In Table S1, we show $\alpha_{n}(n=0,1,2)$ and its limit in comparison with the variationally exact solution of $\alpha$ for decreasing $a$. Their corresponding energies are also tabulated. As one can see, for small enough $a, \alpha_{1}$ is already a good approximation to the true minimizer.

TABLE S1: Comparing the variationally exact solution (minimizer of Eq. (S18)) with the approximate iterative solution of Eqs. (S34)-(S37). Here $E\left(\alpha_{n}\right)$ is evaluated using Eq. (S18), in unit of Hartree.

\begin{tabular}{cc|ccccc}
\hline \hline$a$ (Bohr) & & Var. Exact & $n=0$ & $n=1$ & $n=2$ & $n \rightarrow \infty$ \\
\hline \hline \multirow{2}{*}{$10^{-2}$} & $\alpha$ & 4.025 & 6.056 & 2.454 & 4.261 & 3.534 \\
& $E$ & -14.223 & -12.006 & -12.635 & -14.191 & -14.077 \\
\hline \multirow{2}{*}{$10^{-3}$} & $\alpha$ & 6.999 & 10.661 & 5.928 & 7.102 & 6.821 \\
& $E$ & -37.431 & -29.565 & -36.719 & -37.425 & -37.412 \\
\hline \multirow{2}{*}{$10^{-4}$} & $\alpha$ & 10.598 & 15.266 & 9.815 & 10.698 & 10.553 \\
& $E$ & -76.980 & -64.398 & -76.617 & -76.974 & -76.978 \\
\hline \multirow{2}{*}{$10^{-5}$} & $\alpha$ & 14.529 & 19.871 & 13.893 & 14.609 & 14.520 \\
& $E$ & -134.509 & -118.500 & -134.278 & -134.505 & -134.509 \\
\hline \multirow{2}{*}{$10^{-6}$} & $\alpha$ & 18.629 & 24.477 & 18.081 & 18.687 & 18.627 \\
& $E$ & -210.754 & -191.990 & -210.587 & -210.752 & -210.754 \\
\hline \multirow{2}{*}{$10^{-7}$} & $\alpha$ & 22.826 & 29.082 & 22.342 & 22.869 & 22.826 \\
& $E$ & -306.166 & -285.024 & -306.038 & -306.165 & -306.166 \\
\hline \multirow{2}{*}{$10^{-8}$} & $\alpha$ & 27.089 & 33.687 & 26.653 & 27.121 & 27.089 \\
& $E$ & -421.076 & -397.818 & -420.974 & -421.076 & -421.076 \\
\hline \multirow{2}{*}{$10^{-9}$} & $\alpha$ & 31.399 & 38.292 & 31.002 & 31.424 & 31.399 \\
& $E$ & -555.732 & -530.559 & -555.649 & -555.732 & -555.732 \\
\hline \multirow{2}{*}{$10^{-10}$} & $\alpha$ & 35.744 & 42.897 & 35.380 & 35.765 & 35.744 \\
& $E$ & -710.323 & -683.397 & -710.253 & -710.323 & -710.323 \\
\hline \hline \multirow{2}{*}{$*$} & & & & & &
\end{tabular}


Now let us analyze the asymptotic behavior of the variational energy by substituting series expansion of $\alpha$ into Eq. (S26),

$$
\begin{aligned}
E(\alpha) & =\frac{T(\alpha)-2 K_{0}(2 a \alpha)}{2 a K_{1}(2 a \alpha)}=\frac{T(\alpha)-2 K_{0}(2 a \alpha)}{2 a\left(\frac{1}{2 b}+\mathcal{O}(b \ln b)\right)} \\
& =\alpha \frac{T(\alpha)-2 K_{0}(2 a \alpha)}{1+\mathcal{O}\left(b^{2} \ln b\right)}=\alpha\left(T(\alpha)-2 K_{0}(2 a \alpha)+\mathcal{O}\left(b^{2} \ln b\right)\right) \\
& =\alpha\left[\frac{1}{2} \alpha e^{-2 b}(1+\mathcal{O}(b))+2 \gamma_{0}+2 \ln b+\mathcal{O}\left(b^{2} \ln b\right)\right] \\
& =\alpha\left(\frac{1}{2} \alpha+2 \ln (a \alpha)+2 \gamma_{0}+\mathcal{O}(b)\right) .
\end{aligned}
$$

As a zeroth order approximation,

$$
E_{0}=E\left(\alpha_{0}\right)=\alpha_{0}\left(\frac{1}{2} \alpha_{0}+2 \ln \left(a \alpha_{0}\right)+2 \gamma_{0}\right) .
$$

Let us denote $p=\ln \frac{1}{a^{2}}$. Substituting $\alpha_{0}=p-2-2 \gamma_{0}$ into Eq. (S39), we have

$$
\begin{aligned}
E_{0} & =\left(p-2-2 \gamma_{0}\right)\left(\frac{1}{2} p-p+2 \ln \left(p-2-2 \gamma_{0}\right)+\gamma_{0}-1\right) \\
& =\left(p-2-2 \gamma_{0}\right)\left(-\frac{1}{2} p+\gamma_{0}-1+2 \ln p-\frac{4}{p}\left(1+\gamma_{0}\right)+\mathcal{O}\left(\frac{1}{p^{2}}\right)\right) \\
& =-\frac{1}{2} p^{2}+2 p \ln p+2 \gamma_{0} p-4\left(1+\gamma_{0}\right) \ln p-2\left(1+\gamma_{0}\right)^{2}+\mathcal{O}\left(\frac{1}{p}\right) .
\end{aligned}
$$

Plugging $\alpha_{1}$ into Eq. (S39), we obtain the next order improvement,

$$
E_{1}=E\left(\alpha_{1}\right)=\alpha_{1}\left(\frac{1}{2} \alpha_{1}+2 \ln \left(a \alpha_{1}\right)+2 \gamma_{0}\right) .
$$

Substituting $\alpha_{1}=p-2 \ln p-2-2 \gamma_{0}$ into Eq. (S41), we have

$$
\begin{aligned}
E_{1} & =\left(p-2 \ln p-2-2 \gamma_{0}\right)\left(\frac{1}{2} p-\ln p-p+2 \ln \left(p-2 \ln p-2-2 \gamma_{0}\right)+\gamma_{0}-1\right) \\
& =\left(p-2 \ln p-2-2 \gamma_{0}\right)\left(-\frac{1}{2} p+\gamma_{0}-1+\ln p-\frac{4}{p}\left(\ln p+1+\gamma_{0}\right)+\mathcal{O}\left(\left(\frac{\ln p}{p}\right)^{2}\right)\right) \\
& =-\frac{1}{2} p^{2}+2 p \ln p+2 \gamma_{0} p-2(\ln p)^{2}-4\left(1+\gamma_{0}\right) \ln p-2\left(1+\gamma_{0}\right)^{2}+\mathcal{O}\left(\frac{(\ln p)^{2}}{p}\right) \\
& =\epsilon_{0}+\epsilon_{1}+\epsilon_{2}+\epsilon_{3}+\epsilon_{4}+\epsilon_{5}+\mathcal{O}\left(\frac{(\ln p)^{2}}{p}\right) .
\end{aligned}
$$

Here we have denoted $\epsilon_{0}=-\frac{1}{2} p^{2}, \epsilon_{1}=2 p \ln p, \epsilon_{2}=2 \gamma_{0} p, \epsilon_{3}=-2(\ln p)^{2}, \epsilon_{4}=-4\left(1+\gamma_{0}\right) \ln p$ and $\epsilon_{5}=-2\left(1+\gamma_{0}\right)^{2}$.

In Table S2, we tabulate $\epsilon_{k}(k=0,1, \cdots, 5)$ as $a$ approaches 0 . We also show the error of $\sum_{k=0}^{5} \epsilon_{k}$ with respect to the variationally exact energy $E_{\mathrm{var}}$ and the error of $E_{\mathrm{var}}$ relative to 
the true ground state energy $E_{\text {exact }}$. Here $E_{\text {exact }}$ for $a=10^{-5}$ to $10^{-8}$ are obtained from Ref [10] of the main text. $E_{\text {exact }}$ for smaller $a$ are not available because of numerical challenges. However, from our error trend we can see that $E_{\text {var }}$ is a sufficiently good estimate of $E_{\text {exact }}$. 
TABLE S2: $\epsilon_{k}$ for different $a$. Here the last column shows the difference between the variationally exact energy $E_{\mathrm{var}}$ and the true ground state energy $E_{\text {exact }}$. All energies are in unit of Hartree.

\begin{tabular}{|c|c|c|c|c|c|c|c|c|c|}
\hline$a$ & $\epsilon_{0}$ & $\epsilon_{1}$ & $\epsilon_{2}$ & $\epsilon_{3}$ & $\epsilon_{4}$ & $\epsilon_{5}$ & $\sum \epsilon_{k}$ & $\sum \epsilon_{k}-E_{\mathrm{var}}$ & $E_{\mathrm{var}}-E_{\text {exact }}$ \\
\hline 1.E-01 & -10.60 & 14.07 & 5.32 & -4.66 & -9.63 & -4.98 & -10.50 & -6.71 & 0.03 \\
\hline 1.E-02 & -42.42 & 40.90 & 10.63 & -9.86 & -14.01 & -4.98 & -19.73 & -5.50 & 0.27 \\
\hline 1.E-03 & -95.43 & 72.55 & 15.95 & -13.79 & -16.57 & -4.98 & -42.26 & -4.83 & 0.74 \\
\hline 1.E-04 & -169.66 & 107.34 & 21.27 & -16.98 & -18.38 & -4.98 & -81.39 & -4.41 & 1.09 \\
\hline 1.E-05 & -265.09 & 144.45 & 26.58 & -19.68 & -19.79 & -4.98 & -138.51 & -4.00 & 1.23 \\
\hline 1.E-06 & -381.74 & 183.41 & 31.90 & -22.03 & -20.94 & -4.98 & -214.37 & -3.62 & 1.27 \\
\hline 1.E-07 & -519.59 & 223.92 & 37.21 & -24.12 & -21.91 & -4.98 & -309.46 & -3.30 & 1.28 \\
\hline 1.E-08 & -678.64 & 265.75 & 42.53 & -26.02 & -22.75 & -4.98 & -424.11 & -3.03 & 1.28 \\
\hline 1.E-09 & -858.91 & 308.73 & 47.85 & -27.74 & -23.50 & -4.98 & -558.55 & -2.81 & \\
\hline 1.E-10 & -1060.38 & 352.73 & 53.16 & -29.33 & -24.16 & -4.98 & -712.95 & -2.63 & \\
\hline 1.E-11 & -1283.06 & 397.66 & 58.48 & -30.81 & -24.76 & -4.98 & -887.47 & -2.47 & \\
\hline 1.E-12 & -1526.95 & 443.43 & 63.80 & -32.19 & -25.31 & -4.98 & -1082.20 & -2.33 & \\
\hline 1.E-13 & -1792.04 & 489.97 & 69.11 & -33.49 & -25.82 & -4.98 & -1297.24 & -2.21 & \\
\hline 1.E-14 & -2078.34 & 537.21 & 74.43 & -34.72 & -26.28 & -4.98 & -1532.68 & -2.11 & \\
\hline 1.E-15 & -2385.85 & 585.12 & 79.75 & -35.87 & -26.72 & -4.98 & -1788.56 & -2.01 & \\
\hline 1.E-16 & -2714.57 & 633.64 & 85.06 & -36.98 & -27.13 & -4.98 & -2064.95 & -1.93 & \\
\hline 1.E-17 & -3064.50 & 682.73 & 90.38 & -38.03 & -27.51 & -4.98 & -2361.90 & -1.85 & \\
\hline 1.E-18 & -3435.63 & 732.37 & 95.69 & -39.03 & -27.87 & -4.98 & -2679.44 & -1.78 & \\
\hline 1.E-19 & -3827.97 & 782.52 & 101.01 & -39.99 & -28.21 & -4.98 & -3017.62 & -1.71 & \\
\hline 1.E-20 & -4241.52 & 833.15 & 106.33 & -40.91 & -28.53 & -4.98 & -3376.46 & -1.65 & \\
\hline 1.E-21 & -4676.27 & 884.25 & 111.64 & -41.80 & -28.84 & -4.98 & -3756.00 & -1.60 & \\
\hline 1.E-22 & -5132.24 & 935.78 & 116.96 & -42.66 & -29.14 & -4.98 & -4156.27 & -1.55 & \\
\hline 1.E-23 & -5609.41 & 987.73 & 122.28 & -43.48 & -29.42 & -4.98 & -4577.27 & -1.50 & \\
\hline 1.E-24 & -6107.79 & 1040.08 & 127.59 & -44.28 & -29.68 & -4.98 & -5019.05 & -1.46 & \\
\hline 1.E-25 & -6627.37 & 1092.82 & 132.91 & -45.05 & -29.94 & -4.98 & -5481.61 & -1.42 & \\
\hline 1.E-26 & -7168.17 & 1145.92 & 138.23 & -45.80 & -30.19 & -4.98 & -5964.98 & -1.38 & \\
\hline 1.E-27 & -7730.17 & 1199.38 & 143.54 & -46.52 & -30.43 & -4.98 & -6469.17 & -1.34 & \\
\hline 1.E-28 & -8313.38 & 1253.18 & 148.86 & -47.23 & -30.66 & -4.98 & -6994.19 & -1.31 & \\
\hline 1.E-29 & -8917.79 & 1307.31 & 154.17 & -47.91 & -30.88 & -4.98 & -7540.07 & -1.28 & \\
\hline 1.E-30 & -9543.42 & 1361.76 & 159.49 & -48.58 & -31.09 & -4.98 & -8106.81 & -1.25 & \\
\hline 1.E-31 & -10190.25 & 1416.52 & 164.81 & -49.23 & -31.30 & -4.98 & -8694.43 & -1.22 & \\
\hline 1.E-32 & -10858.29 & 1471.57 & 170.12 & -49.86 & -31.50 & -4.98 & -9302.93 & -1.19 & \\
\hline 1.E-33 & -11547.53 & 1526.91 & 175.44 & -50.47 & -31.69 & -4.98 & -9932.33 & -1.17 & \\
\hline 1.E-34 & -12257.99 & 1582.52 & 180.76 & -51.08 & -31.88 & -4.98 & -10582.64 & -1.14 & \\
\hline 1.E-35 & -12989.65 & 1638.41 & 186.07 & -51.66 & -32.06 & -4.98 & -11253.87 & -1.12 & \\
\hline 1.E-36 & -13742.52 & 1694.57 & 191.39 & -52.24 & -32.24 & -4.98 & -11946.02 & -1.10 & \\
\hline 1.E-37 & -14516.60 & 1750.97 & 196.71 & -52.80 & -32.42 & -4.98 & -12659.11 & -1.07 & \\
\hline 1.E-38 & -15311.88 & 1807.63 & 202.02 & -53.35 & -32.58 & -4.98 & -13393.14 & -1.05 & \\
\hline 1.E-39 & -16128.37 & 1864.53 & 207.34 & -53.89 & -32.75 & -4.98 & -14148.12 & -1.03 & \\
\hline 1.E-40 & -16966.07 & 1921.67 & 212.65 & -54.41 & -32.91 & -4.98 & -14924.05 & -1.02 & \\
\hline
\end{tabular}

